\title{
PROTACs are effective in addressing the platelet toxicity associated with $\mathrm{BCL}-\mathrm{X}_{\mathrm{L}}$ inhibitors
}

\author{
Peiyi Zhang ${ }^{1}$, Xuan Zhang ${ }^{1}\left(\mathbb{D}\right.$, Xingui Liu², Sajid Khan², Daohong Zhou², Guangrong Zheng ${ }^{1 *}$ \\ ${ }^{1}$ Department of Medicinal Chemistry, College of Pharmacy, University of Florida, Gainesville, FL 32610, USA \\ ${ }^{2}$ Department of Pharmacodynamics, College of Pharmacy, University of Florida, Gainesville, FL 32610, USA
}

*Correspondence: Guangrong Zheng, Department of Medicinal Chemistry, College of Pharmacy, University of Florida, Gainesville, FL 32610, USA. zhengg@cop.ufl.edu

Academic Editor: Matthias Baud, University of Southampton, UK

Received: July 13, 2020 Accepted: August 4, 2020 Published: August 31, 2020

Cite this article: Zhang P, Zhang X, Liu X, Khan S, Zhou D, Zheng G. PROTACs are effective in addressing the platelet toxicity

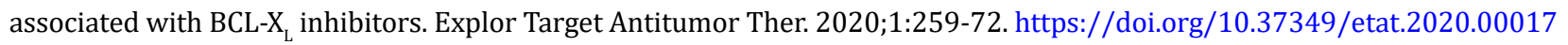

\begin{abstract}
BCL- $\mathrm{X}_{\mathrm{L}}$ is an anti-apoptotic protein that plays an important role in tumorigenesis, metastasis, and intrinsic or therapy-induced cancer drug resistance. More recently, BCL- $\mathrm{X}_{\mathrm{L}}$ has also been identified as a key survival factor in senescent cells. Accumulation of senescent cells has been indicated as a causal factor of aging and many age-related diseases and contributes to tumor relapse and metastasis. Thus, inhibition of BCL- $\mathrm{X}_{\mathrm{L}}$ is an attractive strategy for the treatment of cancer and extension of healthspan. However, development of BCL- $X_{L}$ inhibitors such as navitoclax for clinical use has been challenging because human platelets depend on BCL- $\mathrm{X}_{\mathrm{L}}$ for survival. In this review, the authors discuss how BCL- $\mathrm{X}_{\mathrm{L}}$-targeted proteolysis targeting chimeras (PROTACs) afford a novel approach to mitigate the on-target thrombocytopenia associated with BCL- $\mathrm{X}_{\mathrm{L}}$ inhibition. The authors summarize the progress in the development of BCL-X $\mathrm{X}_{\mathrm{L}}$ PROTACs. The authors highlight the in vitro and in vivo data supporting that by hijacking the ubiquitin protein ligase (E3) that are poorly expressed in human platelets, BCL- $\mathrm{X}_{\mathrm{L}}$ PROTACs can significantly improve the therapeutic window compared to conventional BCL- $\mathrm{X}_{\mathrm{L}}$ inhibitors. These findings demonstrated the potentially broad utility of PROTAC technology to achieve tissue selectivity through recruiting differentially expressed E3 ligases and to reduce on-target toxicity.
\end{abstract}

\section{Keywords}

Apoptosis, BCL- $\mathrm{X}_{\mathrm{L}}$, navitoclax, proteolysis targeting chimera, thrombocytopenia

\section{Introduction}

Apoptosis is a tightly regulated programmed cell death process that plays a vital role in controlling tissue homeostasis. Dysregulation of apoptotic pathways, thereby causes resistance to apoptosis, is a common feature of cancer cells and is also responsible for drug resistance triggered by apoptosis-inducing cancer therapies [1]. Therefore, sensitizing cancer cells to apoptosis is a promising therapeutic strategy for cancer. The B-cell lymphoma 2 (BCL-2) family proteins, consisting of pro- and anti-apoptotic members, are key 
regulators of the intrinsic apoptotic pathway. They control cell apoptosis by modulating the mitochondrial outer membrane permeabilization (MOMP) via protein-protein interactions (PPIs) between the proand anti-apoptotic proteins [2-4]. Anti-apoptotic BCL-2 proteins, including BCL-X, MCL-1, BCL-W, and BFL-1/A1, inhibit MOMP and all have multiple BCL-2 homology (BH) domains. Depending on the structures and the functions they involve in apoptosis regulation, pro-apoptotic BCL-2 proteins can be divided into two subgroups, including BH3-only and multiple BH pore-forming proteins. BH3-only proteins, such as BIK, BIM, BID, BAD, BMF, HRK, NOXA, and PUMA, contain a single BCL-2 BH3 domain, while pore-forming BCL-2 proteins including BAX and BAK contain multiple BH domains. Anti-apoptotic BCL-2 proteins interact with BAX/BAK and prevent them from homo-oligomerization and subsequent pore formation in mitochondrial outer membrane. Upon the stimulation of apoptotic signals, the BH3-only proteins promote apoptosis by directly activating BAX and BAK and/or displacing them from the anti-apoptotic partners. Subsequently, BAK/BAX facilitates MOMP via homo-oligomerization and pore formation in the mitochondrial outer membrane, which causes the efflux of cytochrome $c$ from mitochondria into the cytoplasm. Once released in the cytoplasm, cytochrome $c$ binds with apoptotic protease activating factor-1 (APAF-1), which stimulates caspase 9 to activate the effector caspases and the eventual induction of apoptosis (Figure 1) [5].

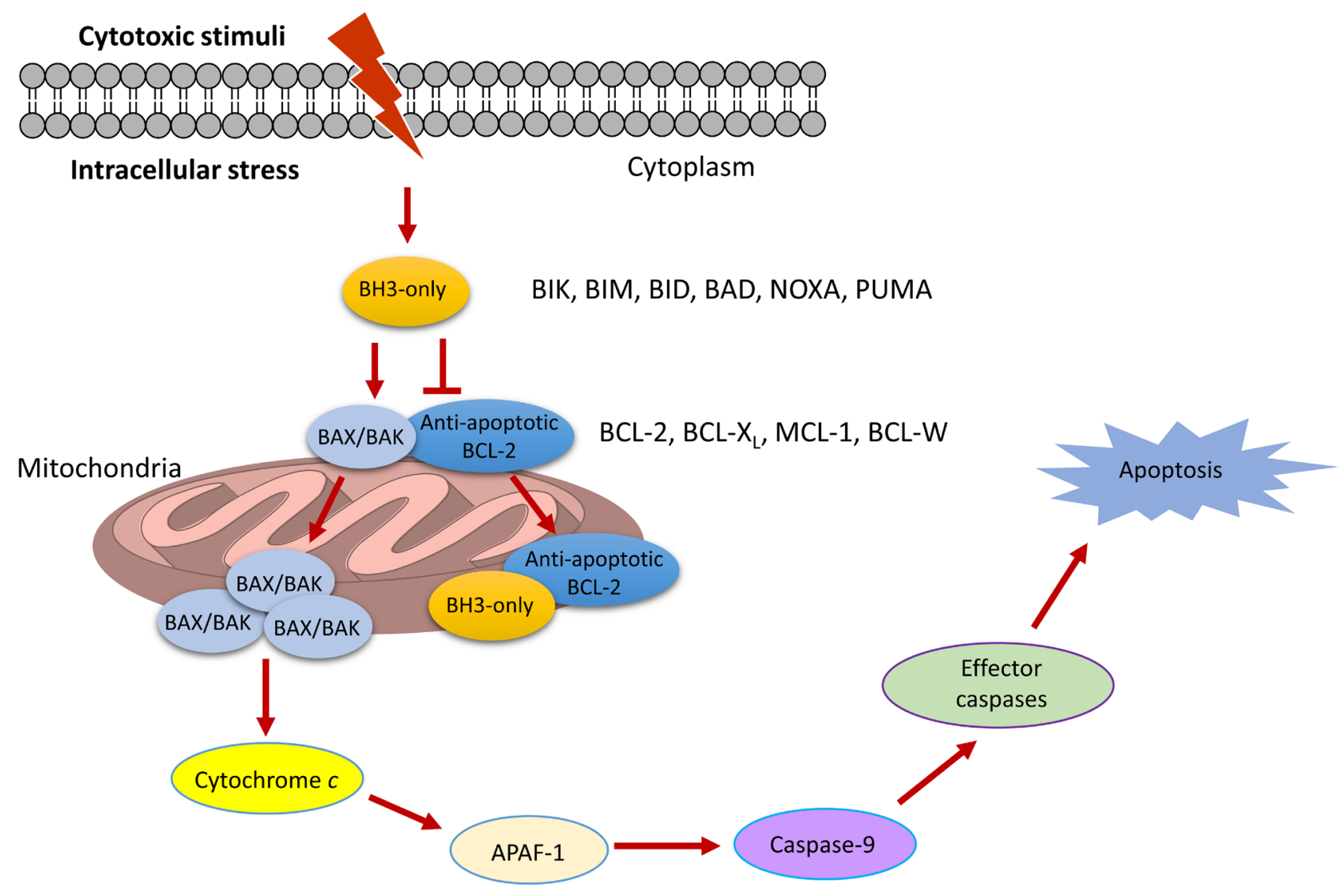

Figure 1. BCL-2 family proteins regulate mitochondria-mediated intrinsic apoptosis pathway

Anti-apoptotic BCL-2 family proteins are upregulated in many cancers and are associated with tumor initiation, progression, and resistance to cancer therapies [6-8]. Thus, inhibiting the PPI between anti- and pro-apoptotic BCL-2 proteins, thereby directly induces apoptosis in cancer cells, is an attractive cancer therapeutic strategy [9-11]. Many "BH3 mimetic" small-molecule inhibitors (SMIs) of the anti-apoptotic BCL-2 proteins, including BCL-2, BCL- $\mathrm{X}_{\mathrm{L}}$, and MCL-1, have been developed [11-13], among which venetoclax (ABT-199, Figure 2) [14], a BCL-2 selective inhibitor, has been approved by the FDA for the treatment of refractory chronic lymphocytic leukemia (CLL) in 2016 [15]. 

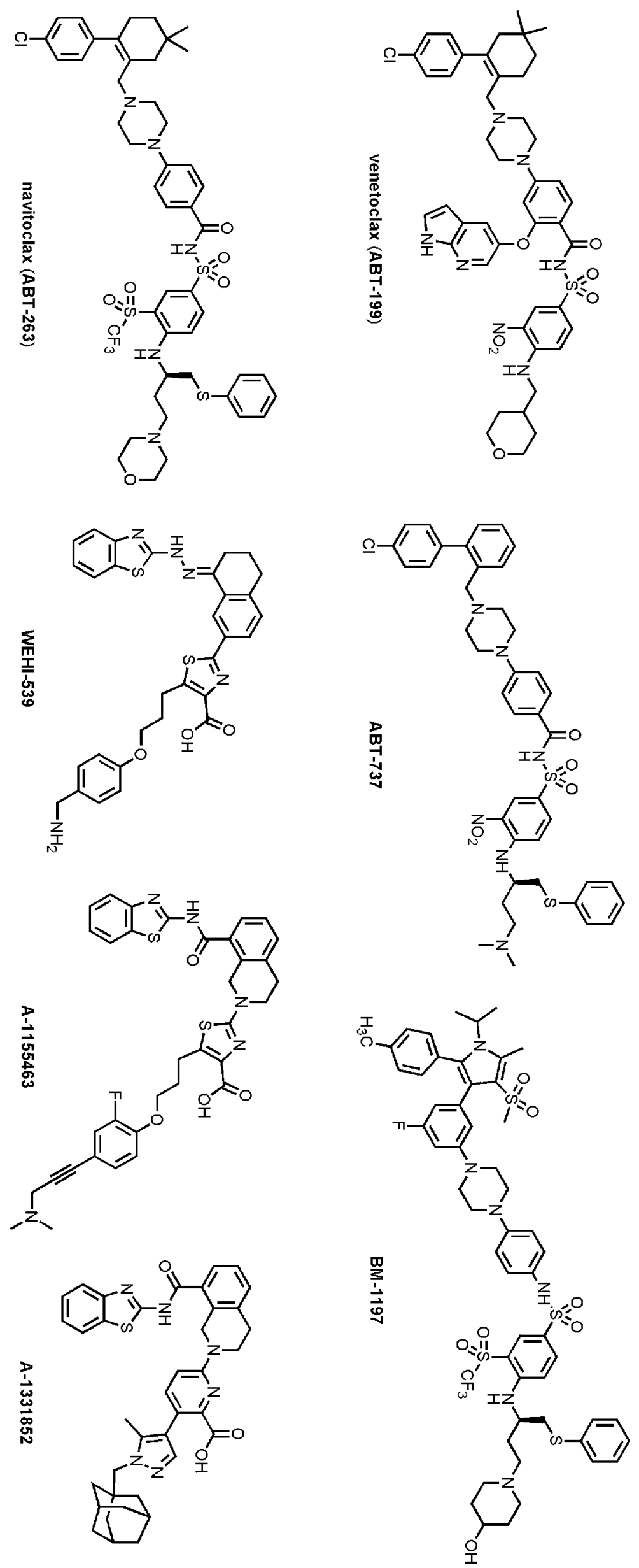

Figure 2. Structures of venetoclax and representative $B C L-X_{L}$ selective (WEHI-539, A-1155463, and A-1331852) and BCL-X $/ B C L-2$ dual inhibitors (ABT-737, BM-1197, and navitoclax) 
Proteolysis targeting chimeras (PROTACs) are heterobifunctional molecules that act by recruiting the E3 ligase to degrade the protein of interest (POI) through the ubiquitin proteasome system. PROTAC technology is a paradigm-shifting approach for targeted protein degradation [16]. The last few years have witnessed rapid progress on this drug discovery platform. PROTAC degraders have been successfully developed for many clinically validated drug targets with two currently in clinical trials for cancer therapy [17]. Several PROTACs that target BCL-2 family proteins have been reported recently [18-21]. This review will summarize the progress on the development of BCL- $\mathrm{X}_{\mathrm{L}}$-targeting PROTACs. We focus on demonstrating the rationale of utilizing PROTAC approach to overcome on-target toxicity. We will also discuss the potential limitations and future directions of this approach.

\section{BCL- $\mathrm{X}_{\mathrm{L}}$ and $\mathrm{BCL}-\mathrm{X}_{\mathrm{L}}$ inhibitors}

The therapeutic strategy of targeting anti-apoptotic BCL-2 proteins has been clinically validated by the FDA approval of venetoclax for the treatment of various hematologic malignancies including CLL and SLL as a single agent, and for AML in combination with low-intensity chemotherapy [22]. However, the complete remission rate $(20 \%)$ of venetoclax-treated CLL patients is relatively low considering the overall response rate is $71-79 \%[23,24]$. This could be attributed to the upregulation of $\mathrm{BCL}-\mathrm{X}_{\mathrm{L}}$ by microenvironmental survival signals [25]. In addition, venetoclax has limited use in solid tumors because BCL-2 upregulation is mainly associated with the survival of hematologic malignancies and is unimportant for the survival of most of the solid tumors $[26,27]$. Across the anti-apoptotic BCL-2 family members, BCL- $\mathrm{X}_{\mathrm{L}}$ is the most frequently overexpressed protein in solid tumors and a subset of leukemia and lymphoma [28]. Tumor resistance to anticancer therapies has also been found to be positively correlated with BCL- $\mathrm{L}_{\mathrm{L}}$ expression [29]. Besides being a cancer target, BCL- $\mathrm{X}_{\mathrm{L}}$ is also essential for the survival of different senescent cells; therefore, BCL- $\mathrm{X}_{\mathrm{L}}$ inhibitors have recently been identified as potent senolytics, i.e. molecules that can kill senescent cells while sparing normal cells [30-32]. As demonstrated in many studies, clearance of senescent cells by senolytics has the potential of treating many age-related diseases and cancer-therapy-induced short-/long-term adverse effects while inhibiting tumor relapse and metastasis [33]. Taken together, $B C L-\mathrm{X}_{\mathrm{L}}$ is one of the most promising therapeutic targets for cancer as well as age-related diseases.

A number of BCL- $X_{L}$ selective or BCL-X $\mathrm{X}_{\mathrm{L}} / \mathrm{BCL}-2$ dual inhibitors have been developed over the past 15 years (Figure 2). ABT-737 was the first potent BCL-X $\mathrm{L}_{\mathrm{L}} / \mathrm{BCL}-2$ dual inhibitor developed via fragment-based drug discovery by NMR approach [34, 35]. Navitoclax (ABT-263) [36] is an orally bioavailable analog of ABT737 and has been in Phase II clinical trials for hematological malignancies and small cell lung cancer (SCLC). However, ABT-263 treatment leads to rapid and dose-dependent thrombocytopenia [37] because platelets depend on $B C L-X_{L}$ to maintain their viability $[38,39]$. Consistently, dose-limiting thrombocytopenia has also been observed in animals treated with other BCL-X ${ }_{L}$ inhibitors, such as ABT-737 [34], BM-1197 [40], and A-1155463 [41]. Thus, thrombocytopenia is an on-target toxicity of BCL- $\mathrm{X}_{\mathrm{L}}$ inhibitors that cannot be solved by conventional medicinal chemistry.

Several strategies have been used to mitigate the on-target thrombocytopenia of BCL- $\mathrm{X}_{\mathrm{L}}$ inhibitors. For example, ABT-263 has been combined with either chemotherapy or targeted therapies so that the platelet toxicity can be manageable due to the reduced clinically effective dose [42, 43]. However, the combination of ABT-263 with docetaxel exacerbated neutropenia in clinical trial, which is believed to be associated with its BCL-2 inhibitory effect [44]. In this case, BCL- $\mathrm{X}_{\mathrm{L}}$ selective inhibitors such as WEHI-539 [45], A-1155463, and A-1331852 [46] (Figure 2) might be more suitable for combination therapy [44]. An alternative strategy is to design prodrugs to minimize drug exposure to human platelets. APG-1252, a phosphate prodrug derived from a BCL-X $\mathrm{X}_{\mathrm{L}}$ BCL-2 dual inhibitor [47], is currently under investigation in Phase I clinical trial (identifier: NCT0308031) [48]. AZD-0466, a dendrimer-conjugated dual BCL-2/BCL-X $\mathrm{L}_{\mathrm{L}}$ inhibitor, has also been shown to have an improved therapeutic index and is currently in phase I clinical trial (identifier: NCT04214093) [49]. Moreover, a BCL- $\mathrm{X}_{\mathrm{L}}$-targeting antibody-drug conjugate (ADC) named ABBV-155 has also entered Phase I clinical trial (identifier: NCT03595059). 


\section{PROTACs}

Since it was initially reported in a proof-of-concept study by Sakamoto et al. [50], in 2001, PROTAC technology has attracted tremendous interests from both industry and academia. PROTACs contain two distinct pharmacophores that are connected via a linker unit; one pharmacophore can bind to the POI and the other recognize a ubiquitin protein ligase (E3). In contrast to SMIs, PROTACs suppress POI in a unique mechanism of action (MOA). In brief, once the PROTAC degrader enters the cell, it forms a ternary complex of POIPROTAC-E3. Subsequent ubiquitin-conjugating enzyme (E2)-involved transfer of ubiquitin results in target polyubiquitination and recycle of the PROTAC into the next round of action. The polyubiquitinated POI can be recognized by $26 \mathrm{~S}$ proteasome and eventually broken into small peptides (Figure 3 ). Various techniques have been developed to characterize each step of this process [51]. Over 50 proteins have been successfully targeted by this technology, including nuclear receptors, protein kinases, epigenetic regulators, neurodegenerative disease-related proteins, regulatory proteins, anti-apoptotic proteins, virus-related proteins, transcription factors, and even E3 ligases [17, 52]. To date, ARV-110 and ARV-471, two PROTAC degraders targeting AR and ER, respectively, have entered Phase I clinical trials (identifiers: NCT03888612, NCT04072952).

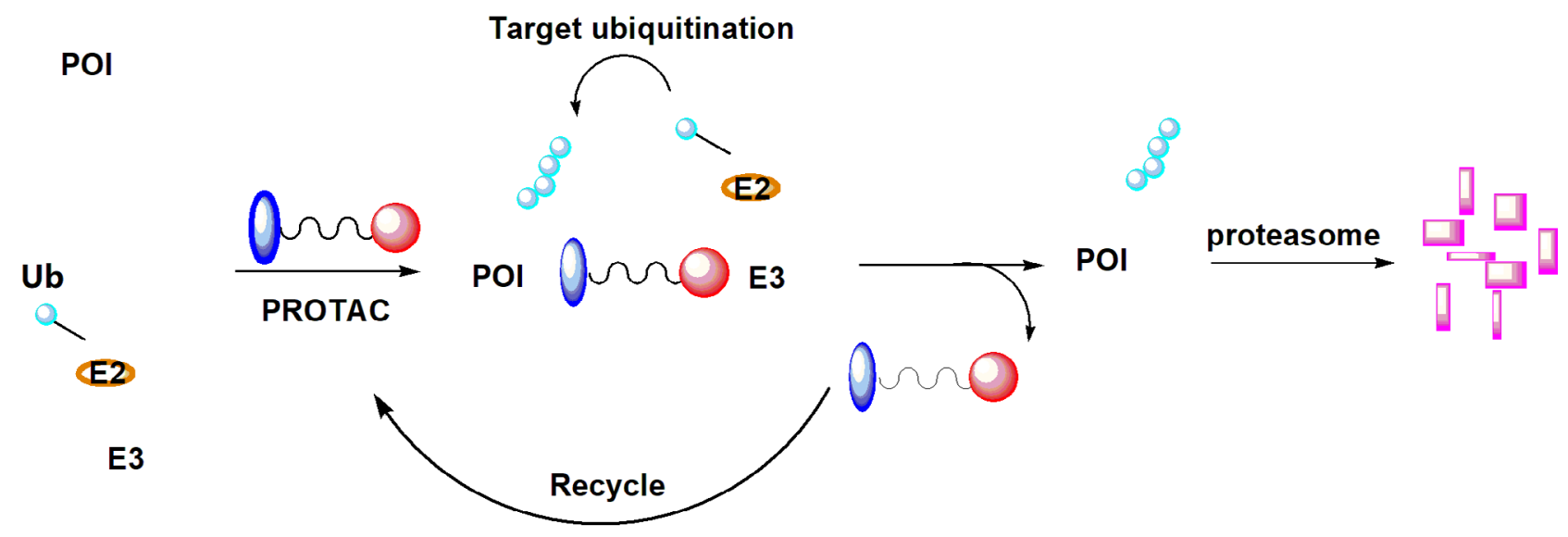

Figure 3. Mechanism of PROTAC-induced POI degradation

Compared with SMIs, PROTACs possess several unique advantages [16]. Due to the catalytic MOA, PROTACs are intrinsically more potent than SMIs. In addition, the warhead, the pharmacophore that binds the POI, of a PROTAC does not need to bind to the active binding site of the POI to achieve protein degradation. Thus, PROTACs hold the potential of eliciting the desired pharmacological effects by targeting the proteins previously considered to be undruggable. Other advantages include the long-lasting suppression of the POI, an added layer of selectivity to further reduce off-target effect, and ability to target mutated proteins.

\section{BCL- $\mathrm{X}_{\mathrm{L}}$-targeting PROTACs}

Efficacy and safety are two major considerations in selecting a drug candidate. Drug toxicity can be due to offtarget or on-target effects. While the off-target toxicity could be addressed by traditional medicinal chemistry approaches, it is extremely challenging to minimize the on-target adverse effect. Besides targeted delivery approaches such as ADC, PROTAC is another potential solution to the on-target toxicity issue. Because PROTACs engage E3 ligases to induce targeted protein degradation, the degradation potency of a PROTAC molecule is also determined by the protein levels of the E3 ligase it recruits. Thus, by recruiting E3 ligases that are relatively higher expressed in the diseased cells/tissues than normal cells/tissues, PROTACs can potentially achieve selective degradation of the POI in the diseased cells/tissues to reduce on-target toxicity (Figure 4).

$\mathrm{BCL}-\mathrm{X}_{\mathrm{L}}$ is an ideal protein for the proof-of-concept study of using PROTAC to overcome the on-target toxicity because thrombocytopenia is known on-target toxicity associated with BCL- $\mathrm{X}_{\mathrm{L}}$ inhibition in platelets $[38,39]$. Our analysis of RNA-sequencing data reveals that all of the commonly used E3 ligases in PROTAC design are expressed at relatively low levels in human platelets [53, 54]. Subsequent western blot assays also suggest that the protein levels of selected E3 ligases along with ubiquitin-activating enzyme (E1) and E2 
enzymes are also low in human platelets compared to different tumor and senescent cells [21]. Thus, based on these initial observations, several series of BCL-X $\mathrm{X}_{\mathrm{L}}$-targeting PROTACs have been designed and synthesized by our group [20, 21, 55-57].

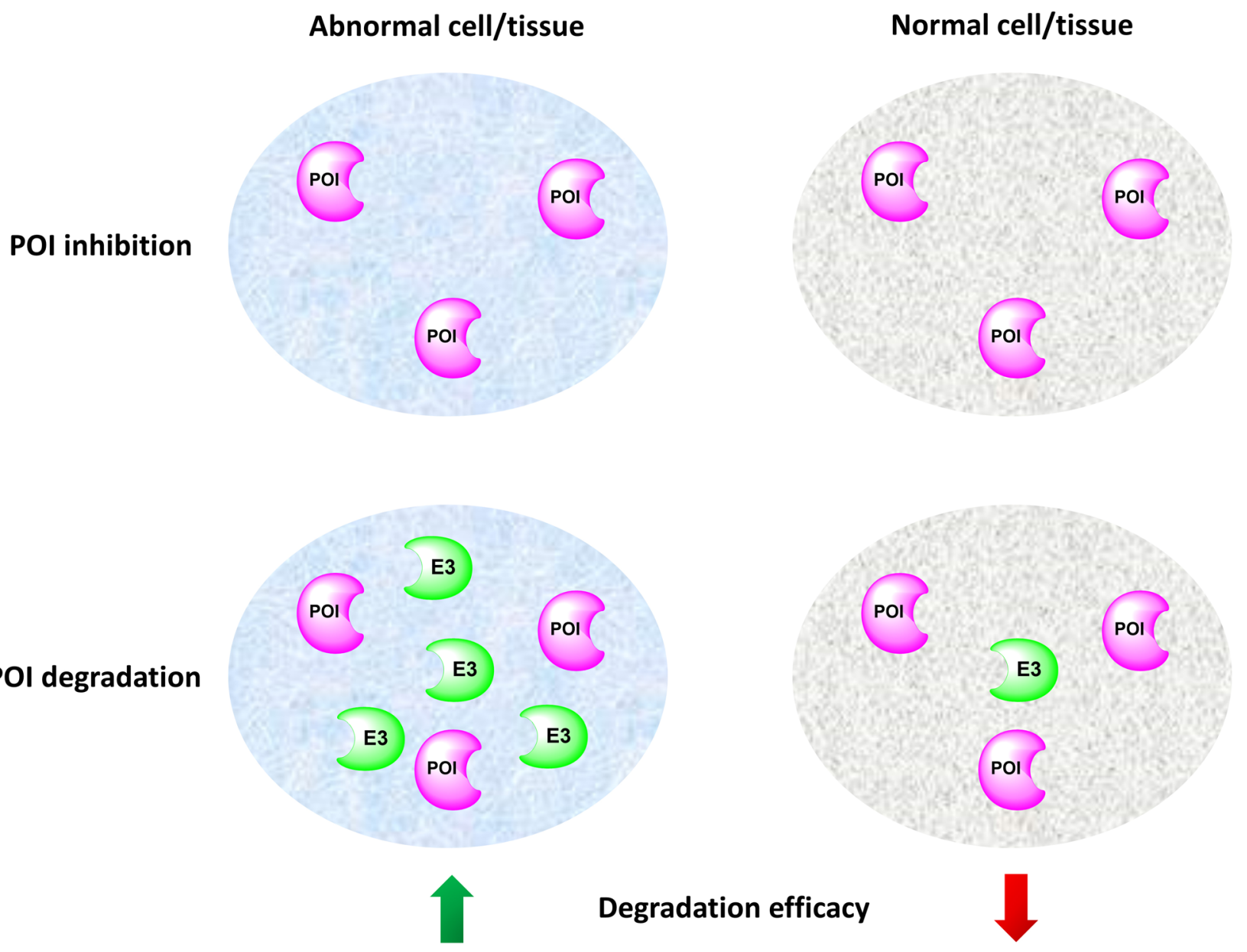

Figure 4. The utilization of PROTAC technology to reduce on-target drug toxicity

DT2216 (Figure 5) is a von Hippel-Landau (VHL)-recruiting PROTAC with the warhead derived from ABT-263. The solvent-exposed morpholine ring in ABT-263 was replaced by a piperazine ring so that a linker can be attached through an amide bond. DT2216 efficiently induced BCL-X $\mathrm{L}_{\mathrm{L}}$ protein degradation in MOLT-4 T-cell acute lymphoblastic leukemia (T-ALL) cells with a half-maximal degradation concentration $\left(\mathrm{DC}_{50}\right)$ of $63 \mathrm{nM}$ and maximum degradation $\left(\mathrm{D}_{\max }\right.$ ) of $90.8 \%$, and the degradation was dependent on both VHL and proteasome. However, only a small reduction of BCL- $\mathrm{X}_{\mathrm{L}}$ levels $\left(\mathrm{D}_{\max }, 26 \%\right)$ was observed in human platelets treated with DT2216 at various concentrations (0.037-3.0 $\mu \mathrm{M})$ [21]. When tested in a cell viability assay, DT2216 was more potent than its parent compound ABT-263 against MOLT-4 cells [half maximal effective concentration $\left(\mathrm{EC}_{50}\right)=52 \mathrm{nM} v s .191 \mathrm{nM}$ ]. Importantly, DT2216 exhibited no toxicity to human platelets up to $3 \mu \mathrm{M}$ concentration while ABT-263 was equally toxic to both MOLT- 4 cells and platelets $\left(\mathrm{EC}_{50}=237 \mathrm{nM}\right)$. The reduced platelet toxicity of DT2216 is likely due to a combination of its minimal induction of BCL- $\mathrm{X}_{\mathrm{L}}$ degradation in platelets, its lower binding affinity to BCL- $\mathrm{X}_{\mathrm{L}}$ and lower cell permeability than ABT-263. Thus, these results provided strong proof-of-concept evidence that converting BCL- $\mathrm{X}_{\mathrm{L}}$ inhibitors to PROTACs can significantly reduce the on-target platelet toxicity of the inhibitors.

Interestingly, although DT2216 had a higher binding affinity to BCL-2 than to BCL-X $\mathrm{X}_{\mathrm{L}}$ Bcl-2 degradation was not observed in all cancer cell lines examined. Proteomics studies also showed that DT2216 specifically reduced BCL- $\mathrm{X}_{\mathrm{L}}$ protein levels [21]. The selective degradation for BCL- $\mathrm{X}_{\mathrm{L}}$ over BCL-2 was also observed with DT2216 analogs in which the VHL-recruiting moiety of DT2216 was replaced by a celebron (CRBN)recruiting moiety [55,56] or inhibitors of apoptosis protein (IAP)-recruiting moiety [57]. It was observed 
that DT2216 could form stable BCL-2-DT2216-VHL ternary complexes in vitro using AlphaLISA assay [58] but not in live cells, as determined by nanoBRET assay [59]. In addition, apparently BCL-2 also lacks a Lys residue in the region where ubiquitin can be transferred to from E2 [21]. These results formed the molecular basis of DT2216 to selectively degrade BCL- $\mathrm{X}_{\mathrm{L}}$, but not BCL-2. This increased specificity can potentially reduce another on-target toxicity of ABT-263, i.e. neutropenia, because neutrophils depend on BCL-2 for survival [60]. Since BCL-2 inhibition exacerbates chemotherapy-induced neutropenia by suppressing granulopoiesis, while inhibition of BCL-X $\mathrm{L}_{\mathrm{L}}$ can sensitize cancer cells to chemotherapy [44], DT2216 has the potential to be combined with chemotherapy for the treatment of different cancers.

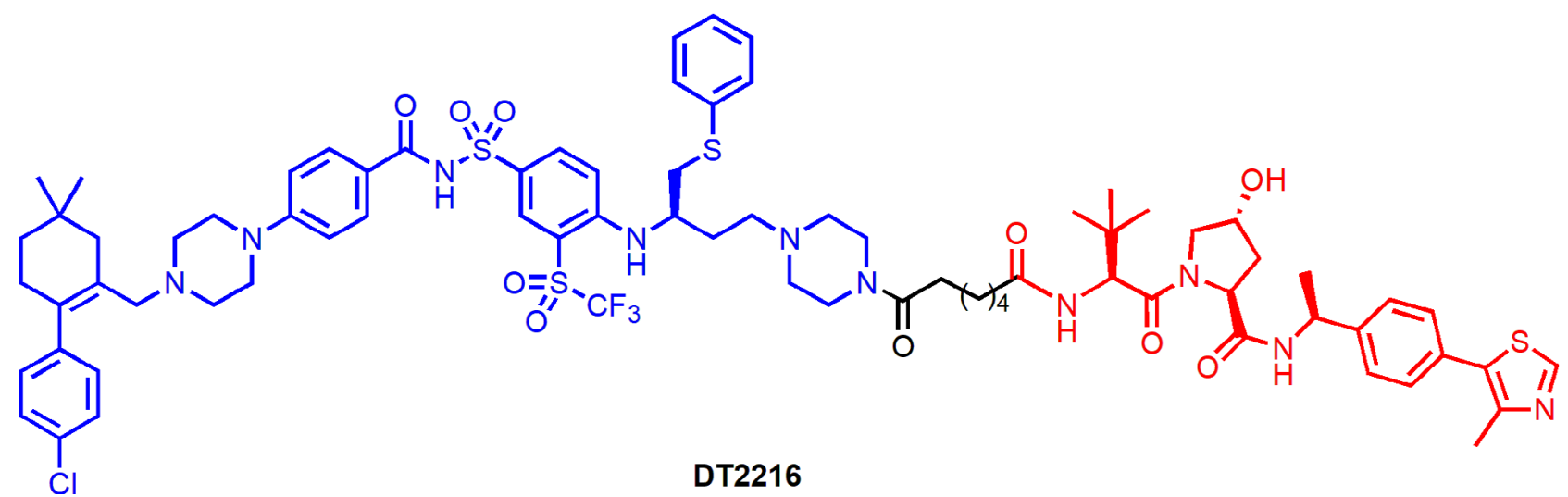

Figure 5. Chemical structure of DT2216 that recruits VHL E3 ligase for BCL- $X_{L}$ degradation

In in vivo antitumor efficacy assays [21], weekly administration of DT2216 at $15 \mathrm{mg} / \mathrm{kg}$ (ip) was more effective in suppressing MOLT-4 T-ALL xenografts in mice than daily treatment with ABT-263 at 50 $\mathrm{mg} / \mathrm{kg}$ (po). The combination of DT2216 (15 mg/kg, q7d, ip) and ABT-199 (50 mg/kg, qd, po) induced nearly complete suppression of tumor growth in mice xenografted with NCI-H146 SCLC cells, which depend on both BCL-X $\mathrm{X}_{\mathrm{L}}$ and BCL-2 for survival. In MDA-MB-231 breast cancer xenograft mouse model, DT2216 (15 mg/kg, $\mathrm{q} 4 \mathrm{~d}$, ip) in combination with docetaxel $(7.5 \mathrm{mg} / \mathrm{kg}$, q14d, iv) was significantly more effective in suppressing the tumor growth than docetaxel alone, indicating that DT2216 can sensitize cancer cells to chemotherapy. In T-ALL PDX models, DT2216 in combination with ABT-199 or chemotherapy (a combination of vincristine $0.15 \mathrm{mg} / \mathrm{kg}$, dexamethasone $5 \mathrm{mg} / \mathrm{kg}$, and L-asparaginase $1,000 \mathrm{U} / \mathrm{kg}$, ip, q7d) exhibited significantly improved anti-leukemic activity in comparison to ABT-199 or chemotherapy alone. In all these studies, severe thrombocytopenia was not observed and DT2216 was found to be much safer than navitoclax on platelets, indicating that DT2216 is a novel anticancer drug candidate that can more safely target BCL- $\mathrm{X}_{\mathrm{L}}$.

A-1155463, a potent BCL- $\mathrm{X}_{\mathrm{L}}$ specific inhibitor, has also been used as the warhead to build CRBNrecruiting PROTACs [20]. CRBN is one of the most frequently E3 ligases used in PROTACs that also shown to lowly expressed in human platelets compared to different tumor cells [20]. The lead compound XZ424 (Figure 6) had similar BCL- $\mathrm{X}_{\mathrm{L}}$ degradation potency $\left(\mathrm{DC}_{50}=50 \mathrm{nM}\right)$ and antiproliferative activity $\left(\mathrm{EC}_{50}=51 \mathrm{nM}\right)$ in MOLT-4 cells when compared with DT2216. XZ424 also showed much lower toxicity to human platelets than its corresponding parent compound A-1155463 $\left(\mathrm{EC}_{50}=1.1 \mu \mathrm{M} v s .7 .1 \mathrm{nM}\right)$. The binding affinity of XZ424 to $\mathrm{BCL}-\mathrm{X}_{\mathrm{L}}$ is similar to A-1155463 [20]. Thus, the reduced platelet toxicity of XZ424 is likely attributable to its minimal induction of BCL- $X_{L}$ degradation and lower cell permeability than A-1155463. Subsequent structural modification led to the discovery of XZ739 (Figure 6), a bioavailable CRBN-recruiting, ABT-263 derived BCL-X degrader with a $\mathrm{DC}_{50}$ of $2.5 \mathrm{nM}$ in MOLT-4 cells [55]. XZ739 is the most potent BCL- $\mathrm{X}_{\mathrm{L}}$ degrader reported and is 20-fold more potent than ABT-263 against MOLT- 4 cells. Notably, DT2216 is less cytotoxic $\left(\mathrm{EC}_{50}=278 \mathrm{nM}\right)$ in NCI-H146 cells whereas XZ739 displayed strong killing effect $\left(\mathrm{EC}_{50}=25 \mathrm{nM}\right)$, indicating XZ739, as a single agent, may have the potential to treat tumors that depend on both BCL- $\mathrm{X}_{\mathrm{L}}$ and BCL-2 for survival. XZ739 also showed good selectivity for MOLT-4 cells over human platelets ( $>100$-fold).

One of the resistance mechanisms of PROTAC-induced degradation is poor protein expression or mutation of the responsible E3 ligase, which could be addressed by the design of PROTACs that recruit 
alternative E3 ligases. PROTAC 1 (Figure 7) is a representative degrader in a series of PROTACs that recruit IAP E3 ligases for BCL- $X_{L}$ degradation. It induced BCL- $\mathrm{X}_{\mathrm{L}}$ degradation and effectively kill malignant MyLa 1929 T-cell lymphoma cells $\left(\mathrm{EC}_{50}=62 \mathrm{nM}\right)$ [57]. Interestingly, CRBN-recruiting PROTACs including XZ424 and XZ739 showed compromised cytotoxicity in this cell line, likely due to the relatively low CRBN expression. PROTAC 1 appeared to be less toxic to human platelets than ABT-263, which can be attributed to the low XIAP expression in platelets according to Western blot analysis [57]. In addition, PROTAC 1 was able to induce robust $\mathrm{BCL}-\mathrm{X}_{\mathrm{L}}$ degradation across multiple cancer cell lines, suggesting that IAP-based degraders targeting $\mathrm{BCL}-\mathrm{X}_{\mathrm{L}}$ hold the potential of extensive applications in cancer treatment.

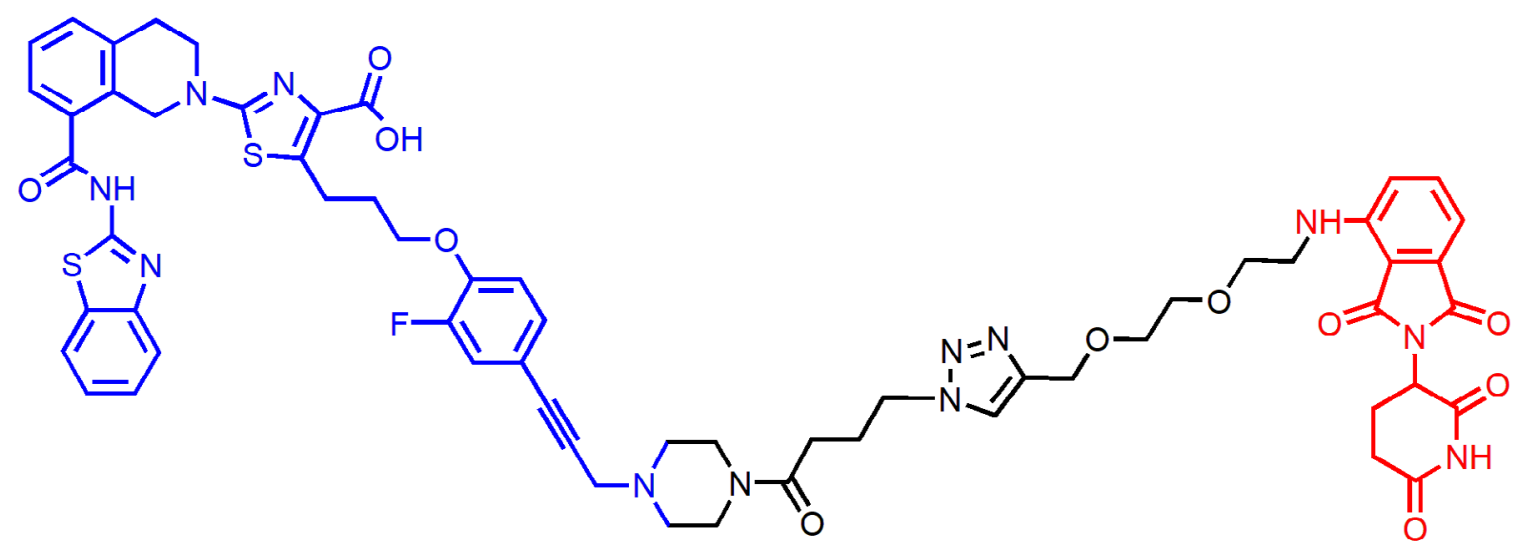

\section{XZ424}<smiles>[Y16][Y16]#N</smiles>

Figure 6. Chemical structures of $X Z 424$ and $X Z 739$ that recruit CRBN E3 ligase for $B C L-X_{L}$ degradation

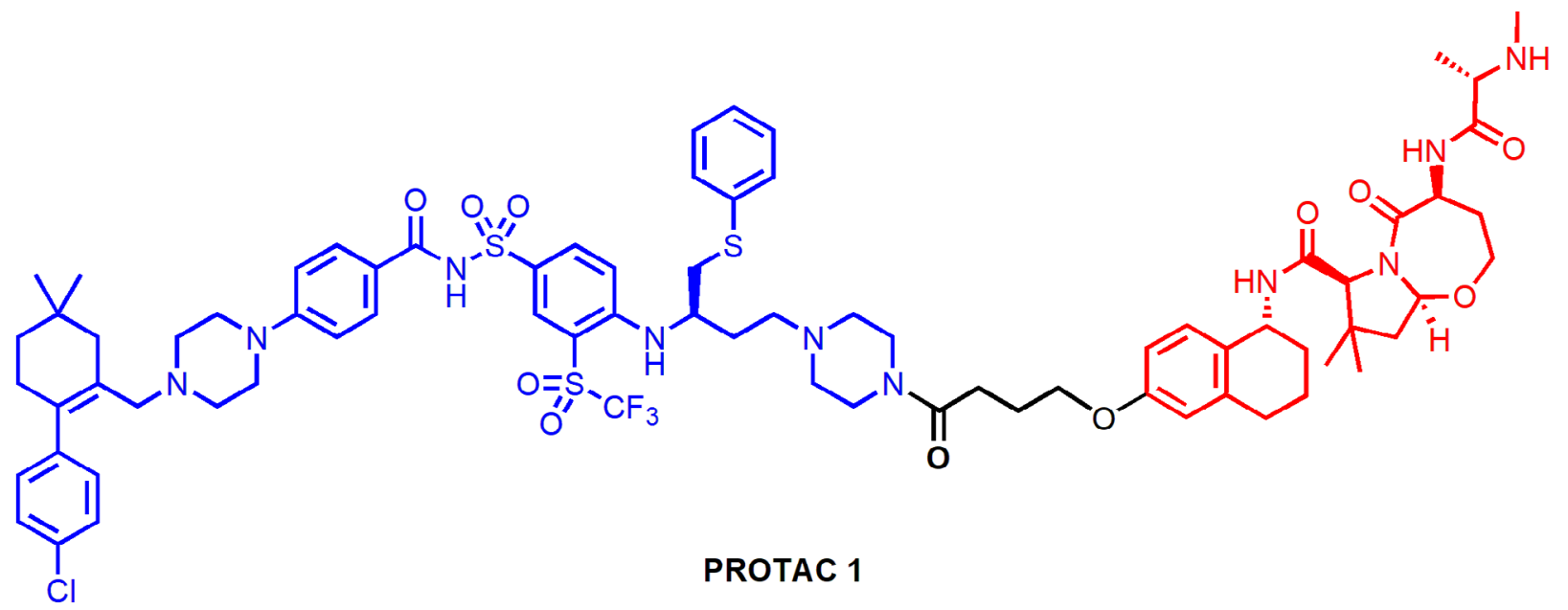

Figure 7. Chemical structure of PROTAC 1 that recruits IAPs for BCL-X $X_{L}$ degradation 
BCL-X $\mathrm{X}_{\mathrm{L}}$ PROTAC degraders have also been tested in senescent cells and in relevant animal models. PZ15227, a CRBN-recruiting degrader derived from ABT-263 (Figure 8), selectively induced BCL-X degradation in senescent cells and non-senescent cells, but not in platelets [56]. Non-senescent cells do not depend on BCL-X $\mathrm{X}_{\mathrm{L}}$ for survival, as a result, PZ15227 selectively induced apoptosis in senescent cells including those derived from WI-38 fibroblasts, IMR90 cells, renal epithelial cells and pre-adipocytes. Overall, compared to ABT263, PZ15227 is much less toxic to human platelets because of low CRBN expression in platelets, but equally or more potent against senescent cells. A galacto-conjugated ABT-263 prodrug, that can be selectively released in senescent cells due to the high levels of $\beta$-galactosidase, has been shown to have improved therapeutic window [61]; however, the cellular activity might be compromised because of the decreased cell permeability and incomplete release of the active drug. In contrast, due to the catalytic MOA, PROTACs have the potential to be more potent than the parent compound.

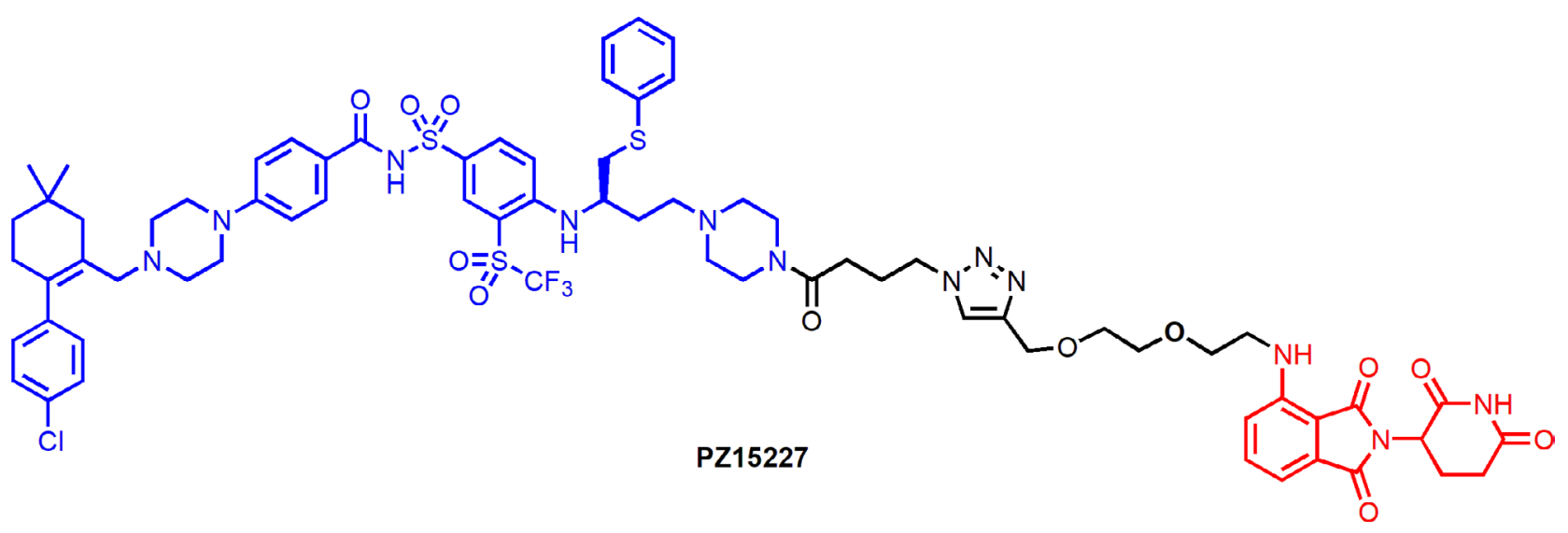

Figure 8. Chemical structure of $P Z 15227$ that recruits $C R B N$ E3 ligase for $B C L-X_{L}$ degradation

In animal studies, PZ15227 (61 mg/kg, q3d, ip) cleared senescent cells without causing notable thrombocytopenia. Moreover, clearance of senescent cells by PZ15227 improved osteoprogenitor functions. Further, PZ15227 attenuated age-related myeloid skewing and rejuvenated hematopoietic stem cells in naturally aged mice.

\section{Conclusion}

BCL- $\mathrm{X}_{\mathrm{L}}$ is one of the most important targets for cancer and senolytic therapy. By converting BCL- $\mathrm{X}_{\mathrm{L}}$ inhibitors to PROTAC degraders, the on-target and dose-limiting thrombocytopenia can be manageable. With further optimization, BCL- $\mathrm{X}_{\mathrm{L}}$ degraders have the potential to achieve extensive clinical applications. It worth noting that Chung et al. [62], recently reported the first crystal structure of VHL-PROTAC-BCL-X $\mathrm{L}_{\mathrm{L}}$ ternary complex, which could be useful for structure-based design of novel BCL- $\mathrm{X}_{\mathrm{L}}$ degraders. However, several potential limitations need to be considered during the investigation. PROTACs may have limited efficacy in tissues that have poor expression levels of the E3 ligase they recruit. The acquired resistance to PROTACs has also been reported after chronic treatment with either CRBN- or VHL-based PROTACs [63]. In addition, some CRBNrecruiting PROTACs have the potential of degrading neo-substrates such as $\mathrm{C} 2 \mathrm{H} 2$ zinc finger proteins [64]. Further, because PROTACs usually have higher molecular weight compared to SMIs, especially for degraders targeting PPI such as the molecules discussed in this review, most of them have high topological polar surface area (TPSA) and hydrophobicity that is far beyond Lipinski's rule-of-five. Compared to SMIs, optimization of the pharmaceutical properties of PROTACs could face more challenges. However, it is still not clear whether PROTACs and SMIs should apply the same set of drug-like physicochemical properties. For example, due to the catalytic mode of action of PROTACs, the requirement for cell membrane permeability might be much lower than SMIs.

PROTAC technology enables us to expand the number of proteins which can be targeted for clinical intervention. Utilization of the differentially expressed E3 ligase to overcome the on-target toxicity is one successful example $[17,65]$. Similar strategy might be useful for other proteins such as another important anti- 
apoptotic protein MCL-1. Among five MCL-1 inhibitors that entered clinical trials, two of those were halted due to "safety signal for cardiac toxicity", which is suspected to be on-target toxicity of MCL-1 inhibition [66]. It will be interesting to investigate if tissue-specific MCL-1 PROTACs can be developed to mitigate cardiac toxicity associated with MCL-1 inhibition.

\section{Abbreviations}

ADC: antibody-drug conjugate

APAF-1: apoptotic protease activating factor-1

BCL-2: B-cell lymphoma 2

BH: BCL-2 homology

CLL: chronic lymphocytic leukemia

CRBN: celeblon

$\mathrm{DC}_{50}$ : half-maximal degradation concentration 50

$\mathrm{D}_{\text {max }}$ : maximum degradation

E2: ubiquitin-conjugating enzyme

E3: ubiquitin protein ligase

$\mathrm{EC}_{50}$ : half maximal effective concentration 50

IAP: inhibitors of apoptosis protein

MOA: mechanism of action

MOMP: mitochondrial outer membrane permeabilization

POI: protein of interest

PPI: protein-protein interaction

PROTAC: proteolysis targeting chimera

SCLC: small cell lung cancer

SMI: small molecule inhibitor

T-ALL: T-cell acute lymphoblastic leukemia

VHL: von Hippel-Landau

XIAP: X-linked inhibitor of apoptosis protein

\section{Declarations}

\section{Author contributions}

PZ and XZ wrote the first draft of the manuscript, and XL and SK wrote additional sections. DZ and GZ revised the manuscript. All authors contributed to manuscript revision and approved the final version.

\section{Conflicts of interest}

The authors declare the following competing financial interest(s): XZ, PZ, SK, DZ, and GZ are co-inventors of the BCL- $\mathrm{X}_{\mathrm{L}}$ PROTACs disclosed in this study. DZ and GZ are co-founders and shareholders of Dialectic Therapeutics, a company that is developing BCL- $\mathrm{K}_{\mathrm{L}}$ PROTACs to treat cancers.

\section{Ethical approval}

Not applicable.

\section{Consent to participate}

Not applicable. 


\section{Consent to publication}

Not applicable.

Availability of data and materials

Not applicable.

\section{Funding}

This work was supported in part by National Institutes of Health grants R21CA223371, R01CA242003, R01AG063801, and R01CA241191. The funder had no role in study design, data collection and analysis, decision to publish, or preparation of the manuscript.

\section{Copyright}

(C) The Author(s) 2020.

\section{References}

1. Igney FH, Krammer PH. Death and anti-death: tumour resistance to apoptosis. Nat Rev Cancer. 2002;2:277-88.

2. Gross A, McDonnell JM, Korsmeyer SJ. BCL-2 family members and the mitochondria in apoptosis. Genes Dev. 1999;13:1899-911.

3. Youle RJ, Strasser A. The BCL-2 protein family: opposing activities that mediate cell death. Nat Rev Mol Cell Biol. 2008;9:47-59.

4. Kale J, Osterlund EJ, Andrews DW. BCL-2 family proteins: changing partners in the dance towards death. Cell Death Differ. 2018;25:65-80.

5. Singh R, Letai A, Sarosiek K. Regulation of apoptosis in health and disease: the balancing act of BCL-2 family proteins. Nat Rev Mol Cell Biol. 2019;20:175-93.

6. Adams JM, Cory S. The Bcl-2 apoptotic switch in cancer development and therapy. Oncogene. 2007;26:1324-37.

7. Reed JC. Bcl-2-family proteins and hematologic malignancies: history and future prospects. Blood. 2008;111:3322-30.

8. Delbridge ARD, Grabow S, Strasser A, Vaux DL. Thirty years of BCL-2: translating cell death discoveries into novel cancer therapies. Nat Rev Cancer. 2016;16:99-109.

9. Thomas S, Quinn BA, Das SK, Dash R, Emdad L, Dasgupta S, et al. Targeting the bcl-2 family for cancer therapy. Expert Opin Ther Targets. 2013;17:61-75.

10. Delbridge AR, Strasser A. The BCL-2 protein family, BH3-mimetics and cancer therapy. Cell Death Differ. 2015;22:1071-80.

11. Opfermann JT. Attacking cancer's Achilles heel: antagonism of antiapoptotic BCL-2 family members. FEBS J. 2016;283:2661-2675.

12. Yap JL, Chen L, Lanning ME, Fletcher S. Expanding the cancer arsenal with targeted therapies: disarmament of the antiapoptotic Bcl-2 proteins by small molecules. J Med Chem. 2017;60:821-38.

13. Zhang X, Liu X, Zhou D, Zheng G. Targeting anti-apoptotic BCL-2 family proteins for cancer treatment. Future Med Chem. 2020;12:563-5.

14. Souers AJ, Leverson JD, Boghaert ER, Ackler SL, Catron ND, Chen J, et al. ABT-199, a potent and selective BCL-2 inhibitor, achieves antitumor activity while sparing platelets. Nat Med. 2013;19:202-8.

15. Deeks ED. Venetoclax: first global approval. Drugs. 2016;76:979-87.

16. Lai AC, Crews CM. Induced protein degradation: an emerging drug discovery paradigm. Nat Rev Drug Discov. 2017;16:101-14. 
17. Khan S, He Y, Zhang X, Yuan Y, Pu S, Kong $Q$ et al. PROteolysis TArgeting Chimeras (PROTACs) as emerging anticancer therapeutics. Oncogene. 2020;39:4909-24.

18. Wang Z, He N, Guo Z, Niu C, Song T, Guo Y, et al. Proteolysis targeting chimeras for the selective degradation of Mcl-1/Bcl-2 derived from nonselective target binding ligands. J Med Chem. 2019;62:8152-63.

19. Papatzimas JW, Gorobets E, Maity R, Muniyat MI, MacCallum JL, Neri P, etal. From inhibition to degradation: targeting the anti-apoptotic protein myeloid cell leukemia 1 (MCL1). J Med Chem. 2019;62:5522-40.

20. Zhang X, Thummuri D, He Y, Liu X, Zhang P, Zhou D, et al. Utilizing PROTAC technology to address the ontarget platelet toxicity associated with inhibition of BCL-X. Chem Commun (Camb). 2019;55:14765-8.

21. Khan S, Zhang X, Lv D, Zhang Q, He Y, Zhang P, et al. A selective BCL-XL PROTAC degrader achieves safe and potent antitumor activity. Nat Med. 2019;25:1938-47.

22. DiNardo CD, Pratz K, Pullarkat V, Jonas BA, Arellano M, Becker PS, et al. Venetoclax combined with decitabine or azacitidine in treatment-naive, elderly patients with acute myeloid leukemia. Blood. 2019;133:7-17.

23. Roberts AW, Davids MS, Pagel JM, Kahl BS, Puvvada SD, Gerecitano JF, et al. Targeting BCL2 with venetoclax in relapsed chronic lymphocytic leukemia. N Engl J Med. 2016;374:311-22.

24. Ashkenazi A, Fairbrother WJ, Leverson JD, Souers AJ. From basic apoptosis discoveries to advanced selective BCL-2 family inhibitors. Nat Rev Drug Discov. 2017;16:273-84.

25. Oppermann S, Ylanko J, Shi Y, Hariharan S, Oakes CC, Brauer PM, et al. High-content screening identifies kinase inhibitors that overcome venetoclax resistance in activated CLL cells. Blood. 2016;128:934-47.

26. Mihalyova J, Jelinek T, Growkova K, Hrdinka M, Simicek M, Hajek R. Venetoclax: a new wave in hematooncology. Exp Hematol. 2018;61:10-25.

27. Perini GF, Ribeiro GN, Pinto Neto JV, Campos LT, Hamerschlak N. BCL-2 as therapeutic target for hematological malignancies. J Hematol Oncol. 2018;11:65.

28. Vogler M. Targeting BCL2-proteins for the treatment of solid tumours. Adv Med. 2014;2014:943648.

29. Amundson SA, Myers TG, Scudiero D, Kitada S, Reed JC, Fornace AJ. An informatics approach identifying markers of chemosensitivity in human cancer cell lines. Cancer Res. 2000;60:6101-10.

30. Chang J, Wang Y, Shao L, Laberge RM, Demaria M, Campisi J, et al. Clearance of senescent cells by ABT263 rejuvenates aged hematopoietic stem cells in mice. Nat Med. 2016;22:78-83.

31. Yosef R, Pilpel N, Tokarsky-Amiel R, Biran A, Ovadya Y, Cohen S, et al. Directed elimination of senescent cells by inhibition of BCL-W and BCL-XL. Nat Commun. 2016;7:11190.

32. Zhu Y, Doornebal EJ, Pirtskhalava T, Giorgadze N, Wentworth M, Fuhrmann-Stroissnigg H, et al. New agents that target senescent cells: the flavone, fisetin, and the BCL- $\mathrm{X}_{\mathrm{L}}$ inhibitors, A1331852 and A1155463. Aging (Albany NY). 2017;9:955-63.

33. Campisi J, Kapahi P, Lithgow GJ, Melov S, Newman JC, Verdin E. From discoveries in ageing research to therapeutics for healthy ageing. Nature. 2019;571:183-92.

34. Oltersdorf T, Elmore SW, Shoemaker AR, Armstrong RC, Augeri DJ, Belli BA, et al. An inhibitor of Bcl-2 family proteins induces regression of solid tumours. Nature. 2005;435:677-81.

35. Bruncko M, Oost TK, Belli BA, Ding H, Joseph MK, Kunzer A, et al. Studies leading to potent, dual inhibitors of Bcl-2 and Bcl-xL. J Med Chem. 2007;50:641-62.

36. Tse C, Shoemaker AR, Adickes J, Anderson MG, Chen J, Jin S, et al. ABT-263: a potent and orally bioavailable Bcl-2 family inhibitor. Cancer Res. 2008;68:3421-8.

37. Wilson WH, O'Connor OA, Czuczman MS, LaCasce AS, Gerecitano JF, Leonard JP, et al. Navitoclax, a targeted high-affinity inhibitor of BCL-2, in lymphoid malignancies: a phase 1 dose-escalation study of safety, pharmacokinetics, pharmacodynamics, and antitumour activity. Lancet Oncol. 2010;11:1149-59.

38. Mason KD, Carpinelli MR, Fletcher JI, Collinge JE, Hilton AA, Ellis S, et al. Programmed anuclear cell death delimits platelet life span. Cell. 2007;128:1173-86. 
39. Zhang H, Nimmer PM, Tahir SK, Chen J, Fryer RM, Hahn KR, et al. Bcl-2 family proteins are essential for platelet survival. Cell Death Differ. 2007;14:943-51.

40. Bai L, Chen J, McEachern D, Liu L, Zhou H, Aguilar A, et al. BM-1197: a novel and specific Bcl-2/Bcl-xL inhibitor inducing complete and long-lasting tumor regression in vivo. PLoS ONE. 2014;9:e99404.

41. Tao ZF, Hasvold L, Wang L, Wang X, Petros AM, Park CH, et al. Discovery of a potent and selective BCL-XL inhibitor with in vivo activity. ACS Med Chem Lett. 2014;5:1088-93.

42. Tolcher AW, LoRusso P, Arzt J, Busman TA, Lian G, Rudersdorf NS, et al. Safety, efficacy, and pharmacokinetics of navitoclax (ABT-263) in combination with erlotinib in patients with advanced solid tumors. Cancer Chemother Pharmacol. 2015;76:1025-32.

43. Chen Q, Song S, Wei S, Liu B, Honjo S, Scott A, et al. ABT-263 induces apoptosis and synergizes with chemotherapy by targeting stemness pathways in esophageal cancer. Oncotarget. 2015;6:25883-96.

44. Leverson JD, Phillips DC, Mitten MJ, Boghaert ER, Diaz D, Tahir SK, et al. Exploiting selective BCL-2 family inhibitors to dissect cell survival dependencies and define improved strategies for cancer therapy. Sci Transl Med. 2015;7:279ra40.

45. Lessene G, Czabotar PE, Sleebs BE, Zobe K, Lowes KN, Adams JM, et al. Structure-guided design of a selective BCL-XL inhibitor. Nat Chem Biol. 2013;9:390-7.

46. Wang L, Doherty GA, Judd AS, Tao ZF, Hansen M, Frey RR, et al. Discovery of A-1331852, a first-in-class, potent, and orally bioavailable BCL-XL inhibitor. ACS Med Chem Lett. 2020;[Epub ahead of print].

47. Bai L, Chen J, Liu L, McEachern D, Aguilar A, Zhou H, et al. BM-1252 (APG-1252): a potent dual specific $\mathrm{Bcl}-2 / \mathrm{Bcl}-\mathrm{xL}$ inhibitor that achieves complete tumor regression with minimal platelet toxicity. Eur J Cancer. 2014;50:109-10.

48. Lakhani NJ, Rasco DW, Tolcher AW, Huang Y, Ji J, Wang H, et al. A phase I study of novel Bcl-2/Bcl-xL inhibitor APG-1252 in patients with advanced SCLC or other solid tumor. J Clin Oncol. 2018;36 Suppl 15:2594.

49. Ramesh P, Medema JP. BCL-2 family deregulation in colorectal cancer: potential for BH3 mimetics in therapy. Apoptosis. 2020;25:305-20.

50. Sakamoto KM, Kim KB, Kumagai A, Mercurio F, Crews CM, Deshaies RJ. Protacs: Chimeric molecules that target proteins to the Skp1-Cullin-F box complex for ubiquitination and degradation. Proc Natl Acad Sci. 2001;98:8554-9.

51. Liu X, Zhang X, Lv D, Yuan Y, Zheng G, Zhou D. Assays and technologies for developing proteolysis targeting chimera degraders. Future Med Chem. 2020;12:1155-79.

52. Burslem GM, Crews CM. Proteolysis-targeting chimeras as therapeutics and tools for biological discovery. Cell. 2020;181:102-14.

53. Bray PF, McKenzie SE, Edelstein LC, Nagalla S, Delgrosso K, Ertel A, et al. The complex transcriptional landscape of the anucleate human platelet. BMC Genomics. 2013;14:1.

54. Kissopoulou A, Jonasson J, Lindahl TL, Osman A. Next generation sequencing analysis of human platelet PolyA+ mRNAs and rRNA-depleted total RNA. PLoS one. 2013;8:e81809.

55. Zhang X, Thummuri D, Liu X, Hu W, Zhang P, Khan S, et al. Discovery of PROTAC BCL-XL degraders as potent anticancer agents with low on-target platelet toxicity. Eur J Med Chem. 2020;192:112186.

56. He Y, Zhang X, Chang J, Kim HN, Zhang P, Wang Y, et al. Using proteolysis targeting chimera technology to reduce navitoclax platelet toxicity and improve its senolytic activity. Nat Commun. 2020;11:1996.

57. Zhang X, He Y, Zhang P, Budamagunta V, Lv D, Thummuri D, et al. Discovery of IAP-Recruiting BCL-XL PROTACs as potent degraders across multiple cancer cell lines. Eur J Med Chem. 2020;199:112397.

58. Gadd MS, Testa A, Lucas X, Chan KH, Chen W, Lamont DJ, et al. Structural basis of PROTAC cooperative recognition for selective protein degradation. Nat Chem Biol. 2017;13:514-21. 
59. Riching KM, Mahan S, Corona CR, McDougall M, Vasta JD, Robers MB, et al. Quantitative live-cell kinetic degradation and mechanistic profiling of PROTAC mode of action. ACS Chem Biol. 2018;13:2758-70.

60. Croker BA, O’Donnell JA, Nowell CJ, Metcalf D, Dewson G, Campbell KJ, et al. Fas-mediated neutrophil apoptosis is accelerated by Bid, Bak, and Bax and inhibited by Bcl-2 and Mcl-1. Proc Natl Acad Sci. 2011;108:13135-40.

61. González-Gualda E, Pàez-Ribes M, Lozano-Torres B, Macias D, Wilson JR 3rd, González-López C, et al. Galacto-conjugation of Navitoclax as an efficient strategy to increase senolytic specificity and reduce platelet toxicity. Aging Cell. 2020;19:e13142.

62. Chung CW, Dai H, Fernandez E, Tinworth CP, Churcher I, Cryan J, et al. Structural insights into PROTACmediated degradation of Bcl-xL. ACS Chem Biol. 2020;[Epub ahead of print].

63. Zhang L, Riley-Gillis B, Vijay P, Shen Y. Acquired resistance to BET-PROTACs (proteolysis-targeting chimeras) caused by genomic alterations in core components of E3 ligase complexes. Mol Cancer Ther. 2019;18:1302-11.

64. Moreau K, Coen M, Zhang AX, Pachl F, Castaldi MP, Dahl G, et al. Proteolysis-targeting chimeras in drug development: a safety perspective. Br J Pharmacol. 2020;177:1709-18.

65. He Y, Khan S, Huo Z, Lv D, Zhang X, Liu X, et al. Proteolysis targeting chimeras are emerging therapeutics for hematologic malignancies. J Hematol Oncol. 2020;13:103.

66. Hird AW, Tron AE. Recent advances in the development of Mcl-1 inhibitors for cancer therapy. Pharmacol Ther. 2019;198:59-67. 